\section{m/S}

médecine/sciences $1989 ; 5: 536-8$

\title{
LA CRYOPRÉSERVATION : UN ART EN EXPANSION QUI SOLLICITE LA RECHERCHE
}

\section{Pierre Douzou}

\section{ADRESSE}

P. Douzou : professeur au Muséum d'Histoire Naturelle. U. 310 Inserm-Inra, Institut de biologie physico-chimique, 13, rue Pierre-etMarie-Curie, 75005 Paris, France.

TIRÉS A PART

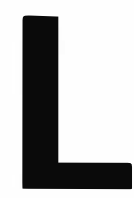

'homme médite depuis longtemps sur le comportement des espèces «à sang froid» hibernant «dans des états ambigus entre la vie et la mort » (J. Rostand). Et comme le froid préserve par ailleurs les tissus morts de la putréfaction, il est logique qu'on ait perçu dans l'avènement des températures cryogéniques un moyen de prolonger quasi indéfiniment la vie de tissus et d'organes isolés, déjà assurée pour un temps limité par du sérum physiologique. En 1912, Alexis Carrel fit ainsi provision de tissus osseux « en vie latente, constamment prêts à être utilisés pour des greffes», mais ce n'est qu'en 1949 que le monde médical redécouvrit les potentialités de la cryopréservation de cellules, tissus et organes perfusés par du glycérol, et dix ans plus tard par du diméthyl sulfoxyde. Le succès quasi immédiat et fortuit dissuada les utilisateurs d'entreprendre l'étude des mécanismes complexes qu'entraîne la congélation de matériaux vivants, et la recherche en la matière se borna le plus souvent à apporter des variantes, des tours de main aux protocoles initiaux, en s'appuyant beaucoup plus sur la physique du changement d'état liquidesolide de l'eau, que sur des considérations biologiques et cytologiques. Souvent pratiquée dans des «officines» mitoyennes de la clinique, la cryopréservation se hissa indûment au rang de discipline et attira nombre de «cryobiologistes » qui produisirent une abondante littérature. Les résultats ne furent pas à la hauteur des espérances, mais permirent tout de même la constitution de banques de sang et de cellules, mais point d'organes pourtant réclamés par les développements de la chirurgie des greffes et des transplantations. Aujourd'hui, la cryopréservation doit faire face à des besoins nouveaux qui dépassent ses possibilités et son cadre d'application initial.

Les progrès fulgurants de la biologie moléculaire et les biotechnologies qui en découlent exigeraient normalement la constitution et la généralisation de «biothèques » où seraient cryopréservées les cellules eucaryotes ou procaryotes génétiquement recombinées.

La fécondation animale et humaine in vitro, la création d'espèces animales et de variétés végétales «transgéniques», imposent à leur tour le stockage cryogénique de gamètes et d'embryons; c'est sous ces mêmes formes que pourrait s'effectuer la conservation des ressources génétiques de la biosphère menacées par le saccage de la biodiversité qui entraîne la disparition de multiples espèces animales et végétales, sauvages et domestiques.

Ainsi la cryopréservation ne peut plus être considérée comme une pratique au service du seul monde médical, et ses carences actuelles nuisent aux 
progrès socio-économiques et humains que l'on attend de la biologie. Devant cette situation, l'état d'esprit, les concepts et les stratégies des tenants de la cryopréservation se sont profondément modifiés durant la dernière décennie. Trop timidement sans doute, mais de façon délibérée, les expérimentations se sont orientées vers une meilleure appréciation des facteurs impliqués dans la congélation des matériaux vivants, avant tout, les conditions de changement d'état liquide-solide de l'eau, qui est le constituant principal de tout le matériel biologique.

Cette eau associée de manière encore mystérieuse à « l'organisation » a été portée «dans tous ses états » possibles : en surfusion, sous ses différentes variétés de glace, additionnée d'antigels tels que le glycérol, l'idée étant que ce sont les microcristaux de glace produits par congélation qui sont responsables des effets létaux de cette dernière, et que tout l'art de la cryopréservation devrait consister à supprimer leur formation.

Il est bien vrai que la formation de cristaux de glace est défavorable à la cryopréservation suivant des mécanismes généraux connus: la congélation des espaces extracellulaires entraîne la formation de fortes concentrations locales en solutés, et l'hypertonicité de ces poches non encore congelées attire l'eau des cellules. La déshydratation prononcée et les chocs osmotiques qu'entraînent ces rapides appels d'eau altèrent irréversiblement l'organisation cellulaire. D'où l'idée, à présent largement répandue, d'obtenir par différents artifices la «vitrification » de l'eau extracellulaire, sous forme de glace amorphe qui prévient l'hyperconcentration des solutés et ses conséquences létales. L'idée est bonne, mais la manière dont elle est exploitée rappelle certains errements antérieurs. Chacun, en effet, recherche ses artifices, parfois constitués de véritables «cocktails » chimiques aux effets secondaires incertains, et toute formule faisant ses preuves sur tel ou tel $m / s n^{\circ} 8$ vol. 5 , octobre 89 matériel est reprise, remaniée empiriquement, dans l'espoir de l'adapter à un nouveau matériel. Il y a encore trop de physique et de physico-chimie, et pas assez de biologie dans cette démarche qui néglige un facteur essentiel : la réponse du cytoplasme à la congélation. En évitant une déshydratation sévère de ce dernier et le choc osmotique qui l'accompagne, on préserve évidemment l'organisation, mais la congélation ultérieure du cytoplasme risque à son tour d'altérer ce dernier par séparation des solutés qui s'aggrègent et de l'eau qui cristallise, annulant ainsi l'effet premier de la vitrification de l'espace extracellulaire. Par ailleurs, on connaît des cellules qui, dûment prétraitées, supportent la formation de cristaux de glace extracellulaire. Des embryons précoces de mammifères (souris, lapin, bovin, homme) et des semences végétales normalement récalcitrants à la congélation deviennent cryotolérants grâce à un protocole qui provoque la déshydratation partielle, puis utilise l'addition de concentrations déterminées de propanediol, polyol auquel les cellules sont perméables. La cryotolérance induite peut atteindre des rendements supérieurs à $80 \%$, et a connu les répercussions importantes que l'on sait en matière de reproduction humaine et de productions animales. Elle offre en outre à l'expérimentateur l'opportunité d'étudier l'effet du prétraitement sur le cytoplasme, et un article publié dans ce numéro (P. Douzou, P. Debey et G. Prulière : «Une nouvelle approche du cryocomportement cellulaire des systèmes vivants ») révèle l'existence d'une corrélation manifeste entre des modifications rhéologiques de l'actine corticale de l'embryon précoce de souris ou de lapin et la cryotolérance observée. Des expériences réalisées sur des mélanges d'actine purifiée et d' $\alpha$-actinine (protéine de liaison) montrent par ailleurs que le prétraitement en question permet de congeler les gels résultants sous forme amorphe (vitrifiée). Enfin, la déshydratation sous vide de ces mêmes gels révèle la difficulté qu'il y a d'extraire l'eau, qui présente ainsi les caractères d'une eau relativement inactive osmotiquement. Ces différentes observations, pour préliminaires qu'elles soient, suggèrent qu'il existerait des situations intracellulaires de nature à favoriser la cryopréservation, via certains réarrangements cytoplasmiques induits par des traitements appropriés. De tels réarrangements pourraient également se manifester spontanément au niveau de cellules d'organismes invertébrés naturellement cryotolérants, alors que leurs fluides corporels sont congelés sous forme cristallisée.

Les expériences réalisées in vitro à partir de solutions d'actine purifiée, en présence d' $\alpha$-actinine et de propanediol sont particulièrement intéressantes. Elles permettent d'obtenir des gels qui se révèlent très homogènes et microporeux à l'examen microscopique, puis se transforment en solides cohérents amorphes par congélation et par déshydratation.

Ce résultat inhabituel rapproche le procédé (dérivé, comme on a vu, du protocole assurant la cryopréservation de l'embryon) des « traitements sol-gel » qui, à partir d'oxydes métalliques en solution et d'agents chimiques appropriés dont, c'est à noter, le glycérol et la formamide, permettent aujourd'hui de fabriquer des verres et des céramiques. Une incursion dans ce domaine peu familier au biologiste m'a convaincu du parallélisme étroit des traitements de l'actine et de composés inorganiques, via des transformations sol-gel de nature séquentielle et contrôlées par des polyols. Différentes protéines d'origine animale et végétale ont été soumises au laboratoire à de tels traitements et ont déjà produit tout une gamme de solides, aux propriétés variées, dérivant tous de gels microporeux et isotropes, c'est-à-dire dont la structure est homogène dans les trois dimensions de l'espace.

Même s'il est prématuré de pousser 
trop loin la comparaison entre les matrices cytoplasmiques cryotolérantes et ces nouveaux matériaux, on peut observer que le cytoplasme n'est, après tout, qu'un vaste réseau de protéines fibrillaires et globulaires en interaction entre elles et avec l'eau et susceptibles de constituer des entités structurales analogues aux gels précurseurs de matériaux composites. A l'examen plus approfondi, il apparaît même comme une mosaïque de « nanocomposites » dont les dimensions varient entre 10 et 100 nanomètres, semblables en cela à des matériaux récemment développés et impliquant des composés inorganiques, organiques et des polymères à longue chaîne. La différence essentielle entre ces formes de matière et celles qui résultent du travail des céramistes réside dans l'instabilité foncière des premières, sans doute nécessaire à la vie cellulaire qui suppose une rhéologie cytoplasmique incompatible avec des structures figées.

Du fait de l'extrême hétérogénéité de composition et des rapports particuliers des biomolécules avec l'eau, l'entité structurale des biocomposites cytoplasmiques ne résiste pas aux contraintes thermiques, de cisaillement, d'évaporation, ou simplement d'altérations physico-chimiques de milieu. Est-il possible d'envisager des phénomènes de décomposition et de recomposition des biomatériaux susceptibles d'homogénéiser leur texture et de la protéger contre ces altérations? C'est une hypothèse que l'on peut proposer au cryobiologiste comme au cytologiste.

A défaut de donner des solutions rapides et générales, sa vérification à partir de concepts et de traitements empruntés à la science des matériaux peut ouvrir de nouvelles perspectives à la connaissance des propriétés rhéologiques du cytoplasme, et à l'étude de la cryopréservation. Cette dernière exige d'être traitée par une recherche pluridisciplinaire, et non comme un Art dont la pratique a atteint très tôt des limites incompatibles avec l'expansion requise par les progrès et les perspectives de la biologie et de ses principales applications 